\title{
Bacteriophages-Versatile Tools of The Past and The Future
}

\author{
Costin Damian ${ }^{1}$, Ramona Gabriela Ursu ${ }^{2}$ and Luminița Smaranda Iancu ${ }^{1 *}$ \\ ${ }^{1}$ Grigore T Popa, University of Medicine and Pharmacy Iași-student, Europe \\ ${ }^{2}$ Grigore T Popa, University of Medicine and Pharmacy Iași-Department of Preventive Medicine and Interdisciplinarity, Europe
}

*Corresponding author: Luminița Smaranda, Grigore T Popa, University of Medicine and Pharmacy Iași, Europe

To Cite This Article: Costin Damian, Ramona Gabriela Ursu, Luminița Smaranda Iancu. Bacteriophages-Versatile Tools of The Past and The Future. Am J Biomed Sci \& Res. 2021 - 13(3). AJBSR.MS.ID.001870. DOI: 10.34297/AJBSR.2021.13.001870.

Received: 阱 March 29, 2021; Published: 製 June 28, 2021

\begin{abstract}
Bacteriophages are ubiquitous viruses which infect bacteria. They have been employed in studies that helped us understand the replication of viruses, their genetics, the structure of DNA and RNA and numerous other concepts that are now staples of modern biomedical sciences. Due to the need of finding an effective treatment against infectious diseases, phages were studied and tried as a treatment, but such prospects have gradually been abandoned as antibiotics became available. They have been used as a model organism of molecular biology, and phage therapy has gained renewed interest since the beginning of the antimicrobial resistance crisis. Phages have the potential to become narrow-spectrum modulators of the microbiome, eliminating only pathogenic species of bacteria, without causing dysbiosis, which is a concept now linked to numerous diseases. Other important applications of phages include phage display, the creation of different vaccines against pathogens or cancerous cells or as drugdelivery agents. Successful use as experimental, compassionate therapy has led to some promising results, but many challenges must be met before it becomes a standardized, largely applied therapy. Still, prospects of using phages in nanotechnology and synthetic biology hold great promise in keeping phages in the spotlight of research.
\end{abstract}

Keywords: Bacteriophage; Phage; Antimicrobial Resistance; Phage Therapy; Vaccine; Lysins; Infect Bacteria; Viruses; Coronavirus; Genetics; Pandemic; Medical Sciences

\section{Introduction}

Bacteriophages (colloquially named phages) are viruses that infect microorganisms belonging to the domain Bacteria. Those viruses are found in abundance everywhere bacteria thrive, being one of the most widespread biological entities on Earth, their number being approximated to $10^{31}$ phages [1]. Their remarkable ubiquity and diversity make them one of the most studied organisms, and these studies lead to solving many unanswered questions of microbiology, molecular biology, and genetics [2]. Infectious diseases have always been a great burden on human health, as everyone can presently experience during the Coronavirus Disease 2019 (Covid-19) pandemic, and medical sciences tried to find treatments to combat those diseases. Before the widespread availability of antibiotics, phage therapy has been considered a viable means of treating infectious diseases, with early animal model trials taking place as early as 1919 [3], and news of the possible cure for infections has spread to different parts of the world, including the former Soviet Union and Eastern Europe [4], Brazil [5], India [6], and even in the United States [7], though for a short time. After penicillin became available in 1943 and up to 1980, phages have been employed by science in numerous studies regarding viral biology and replication, mutations, genetic recombination, transduction, the structure of DNA and RNA, and protein synthesis [8].

Other scientific breakthroughs involving phages include phage display [9] and CRISPR (Clustered Regularly Interspaced Short Palindromic Repeats) [10]. Phage therapy for bacterial infections, however, has fallen to the wayside of research in Western countries due to the efficiency of antibiotics, being continued only in the former communist bloc [11]. Renewed interest in phage therapy has been on the rise since the beginning of the antimicrobial resistance crisis, one of the greatest threats to human life, which is expected to be the cause of 10 million deaths per year by 2050 , 
surpassing deaths related to cancer [12]. The search for new antibiotics to combat resistance is a race against time in which we seem to be lagging, due to new antibiotic discoveries being in decline, because such research has become prohibitively expensive [13]. Thus, trying to find alternative ways to tackle the problem of antibiotic resistance has become a significant topic of research. Much progress has been done in understanding how phages may be used as therapeutic agents, since the days of the early pioneers, as we now understand enough of the biology of phages to allow us to use them safely and effectively. We will discuss some of their properties and how they can be used in therapy's advantage.

Phages are highly specific pathogens, being able to infect only a small number of related bacterial strains, only very few identified phages being able to infect more than one species [14]. This means that phages can be viewed as a narrow-spectrum therapeutic agent, useful when targeting a known bacterial pathogen. However, this means that highly accurate microbiological diagnostic of the etiology of the infection is needed, which may be too expensive or challenging in some parts of the world. Modern understanding of the importance of the microbiome* (that is, all the microorganisms that colonize the human body) in health and disease has turned the biomedical community's attention towards these kinds of narrow-spectrum therapeutic agents. Numerous recent findings about the microbiome now link its alteration to several diseases, such as inflammatory bowel disease, rheumatic arthritis, obesity, diabetes, neurodegenerative disorders, and cancer [15]. The use of broad-spectrum antibiotics can greatly alter the microbiome, which can lead to further decline of critically ill patients [16]. Also, these patients could already receive other treatments for their comorbidities which may interact with antibiotics or amplify the medication's side effects. For reserve antibiotics, employed in treating multidrug-resistant bacteria, such as colistin, these side effects can be very significant [17].

Treatment failure of some multidrug-resistant bacterial infections, even when using reserve antibiotics, has led to multiple instances of using experimental phage therapy for compassionate use, which is a complex endeavor, as multiple criteria must be met and adequate phage must be discovered, tested, or sourced from producers where phage therapy is better established, such as Eastern European countries [18]. Multiple successful compassionate use therapy case reports have been published, including several infections caused by priority pathogens (as they are defined by the World Health Organization) [19]. Some notable examples of successful compassionate use of phage therapy include two successfully treated carbapenem-resistant Acinetobacter baumannii infections in critically ill Covid-19 patients [20], a multidrug-resistant Pseudomonas aeruginosa infection of an aortic graft [21], an Extended-Spectrum Beta-Lactamase Escherichia coli (ESBL) urinary tract infection [22] and a methicillin-resistant Staphylococcus aureus osteomyelitis [22]. An alternative to administering phage in the treatment of bacterial infections is the utilization of phage antimicrobial products, called lysins, which are enzymes that degrade the bacterial cell wall during lytic phage release. Gram-positive bacteria are more susceptible to these compounds because their cell wall is externally accessible, allowing for lysins to work from the outside of the bacteria [23].

Another way phages can be used against diseases is by utilizing them in the creation of vaccines, through the technique named phage display. Some phages can be employed in the development of vaccines against parasites, fungi, anthrax, plague, foot and mouth disease, Epstein-Barr virus, hepatitis B and Zika viruses. Oncologic patients can benefit from phage-based anti-cancer vaccines as well, which target tumor-specific antigens of breast cancer, melanoma, lung cancer and liver cancer cells [24]. A novel breakthrough in oncology involves phage-based nanotechnology in fighting multiple cancer promoting factors at the same time. Some bacteria are commonly found associated with cancer cells, even in distant metastases, which have been named Tumor-Associated Bacteria (TAB) and are proven to exhibit pro-tumoral mechanisms [25]. These bacteria can be specifically eradicated using modified phages, that at the same time deliver chemotherapy to the site of the tumor and release substances that increase the number of bacterial species exhibiting anti-tumoral properties (such as butyrate-producing bacteria) [26]. The future application of phages in biomedical sciences can be considered a certainty, as they have contributed significantly to the advance of science in the past. Whether phage therapy will be a successful solution in fighting against antimicrobial resistance seems promising, but numerous obstacles will have to be overcome before it is largely applied.

\section{Acknowledgement}

None

\section{Conflict of interest}

No Conflict of interest

\section{References}

1. Mushegian AR (2020) Are there 1031 virus particles on earth, or more, or fewer? J bacteriol 202(9).

2. Ofir G, Sorek R (2018) Contemporary phage biology: from classic models to new insights. Cell 172(6): 1260-1270.

3. Letarov AV (2020) History of Early Bacteriophage Research and Emergence of Key Concepts in Virology. Biochemistry (Moscow) 85(9): 1093-1112.

4. Gelman D, Eisenkraf A, Chanishvili N, Nachman D, Glazer SC, et al. (2018) The history and promising future of phage therapy in the military service. J Trauma Acute Care Surg 85(1S): S18-S26.

5. De Freitas Almeida GM, Sundberg LR (2020) The forgotten tale of Brazilian phage therapy. Lancet Infectious Diseases 20(5): e90-e101. 
6. Summers WC (1993) Cholera and plague in India: the bacteriophage inquiry of 1927-1936. J history med allied sci 48(3): 275-301.

7. Summers WC (2001) Bacteriophage therapy. Annual Rev Microbiol 55(1): 437-451.

8. Morange M (2020) The black box of biology: a history of the molecular revolution. Harvard University Press, USA.

9. Hentrich C, Ylera F, Frisch C, Ten Haaf A, Knappik A (2018) Monoclonal Antibody Generation by Phage Display: History, State-of-the-Art, and Future. Handbook of Immunoassay Technologies pp.47-80.

10. Ishino Y, Krupovic M, Forterre P (2018) History of CRISPR-Cas from encounter with a mysterious repeated sequence to genome editing technology. J bacteriol 200(7): e00580-17.

11. Myelnikov D (2018) An alternative cure: the adoption and survival of bacteriophage therapy in the USSR, 1922-1955. J history med allied sci 73(4): 385-411.

12. Bassetti M, Poulakou G, Ruppe E, Bouza E, Van Hal SJ, et al. (2017) Antimicrobial resistance in the next 30 years, humankind, bugs, and drugs: a visionary approach. Intensive care med 43(10): 1464-1475.

13. Renwick M, Mossialos E (2018) What are the economic barriers of antibiotic R\&D and how can we overcome them? Expert opinion on drug discovery 13(10): 889-892.

14. Koskella B, Meaden S (2013) Understanding bacteriophage specificity in natural microbial communities. Viruses 5(3): 806-823.

15. Zheng D, Liwinski T, Elinav E (2020) Interaction between microbiota and immunity in health and disease. Cell research 30(6) 492-506.

16. Bhalodi AA, van Engelen TS, Virk HS, Wiersinga WJ (2019) Impact of antimicrobial therapy on the gut microbiome. J Antimicrob Chemotherapy 74(Sup_1): i6-i15.
17. Petrosillo N, Taglietti F, Granata G (2019) Treatment options for colistin resistant Klebsiella pneumoniae: present and future. J clin med 8(7): 934.

18. McCallin S, Sacher JC, Zheng J, Chan BK (2019) Current state of compassionate phage therapy. Viruses 11(4): 343.

19. World Health Organization (2017) WHO publishes list of bacteria for which new antibiotics are urgently needed? Saudi Med J 38(4): 444-445.

20. Wu N, Dai J, Guo M, Li J, Zhou X, et al. (2021) Pre optimized phage therapy on secondary Acinetobacter baumannii infection in four critical COVID-19 patients. Emerg Microb Infect 10(1): 612-618.

21. Chan BK, Turner PE, Kim S, Mojibian HR, Elefteriades JA, et al. (2018) Phage treatment of an aortic graft infected with Pseudomonas aeruginosa. Evoln med public health 2018(1): 60-66.

22. Patey O, McCallin S, Mazure H, Liddle M, Smithyman A, et al. (2019) Clinical indications and compassionate use of phage therapy: personal experience and literature review with a focus on osteoarticular infections. Viruses 11(1): 18.

23. Heselpoth RD, Swift SM, Linden SB, Mitchell MS, Nelson DC (2021) Enzybiotics: endolysins and bacteriocins. Bacteriophages: Biology, Technology Therapy pp.989-1030.

24. Hess KL, Jewell CM (2020) Phage display as a tool for vaccine and immunotherapy development. Bioeng transl med 5(1): e10142.

25. Song W, Anselmo AC, Huang L (2019) Nanotechnology intervention of the microbiome for cancer therapy. Nat nanotechnol 14(12): 1093-1103.

26. Zheng DW, Dong X, Pan P, Chen KW, Fan JX, et al. (2019) Phage-guided modulation of the gut microbiota of mouse models of colorectal cancer augments their responses to chemotherapy. Nat biomed eng 3(9): 717728. 